\title{
Observation of Dislocation-Assisted 2-Dimensional Conductive Channels Embedded in Perovskite Thin Films
}

\author{
Huaixun Huyan ${ }^{1}$, Linze Li ${ }^{1}$, Yi Zhang ${ }^{1}$, Colin Heikes ${ }^{4}$, Darrel Schlom ${ }^{4}$, Xiaoqing Pan ${ }^{1,3,4 *}$ \\ 1. Department of Materials Science and Engineering, University of California - Irvine, Irvine, CA, USA. \\ 2. Department of Physics and Astronomy, University of California - Irvine, Irvine, CA, USA. \\ 3. Irvine Materials Research Institute, University of California - Irvine, Irvine, CA, USA. \\ 4. Department of Materials Science and Engineering, Cornell University, Ithaca, NY, USA. \\ * Corresponding author: xiaoqingp@uci.edu
}

Fabrication of 1-Dimentional (1D) or 2-Dimentional (2D) nanochannels into a material matrix opens a path to controllable fabrication of complex nanostructures with refined patterns and allows the design strategy for novel and flexible nanodevices. During the growth of crystalline structures, various defects can form, which in turn may affect the growth dynamics and result in unique microstructures in nanoscale. Recently, it was found that dislocations, one of the most common types of defects, can cause the formation of arrays of metallic Ti nanowires into sapphires ${ }^{1}$, or induce the growth of superlattices of $\mathrm{WS}_{2}$ or $\mathrm{MoS}_{2}$ nanoribbons into $\mathrm{WSe}_{2}$ monolayers ${ }^{2,3}$, which represents the self-assembling of patterned quasi-1D channels into 2D or 3D matrices. However, the capability of producing ordered patterns of 2D channels into 3D materials have yet to be explored. In this work, we report the observation of the dislocationassisted $\sim 1$ nm-thick 2D $\mathrm{BiMnO}_{3}$ nanochannels in bulk perovskite thin films, the structure and composition are studied using cross-sectional scanning transmission electron microscopy (STEM), atomic energy dispersive X-ray spectroscopy (EDS), and conductive atomic force microscopy (c-AFM).

The thin film was synthesized by growing 25 u.c. thick $\mathrm{SrTiO} 3$ on 50 u.c. $\mathrm{La}_{0.7} \mathrm{Sr}_{0.3} \mathrm{MnO}_{3}$ on $\mathrm{TbScO}_{3}$ substrate. Then the Bi flux was kept on for $100 \mathrm{~nm} \mathrm{Bi} \mathrm{equivalent} \mathrm{growth} \mathrm{time} \mathrm{to} \mathrm{deposit} \mathrm{a} \mathrm{Bi} \mathrm{layer} \mathrm{on} \mathrm{top}$ of $\mathrm{SrTiO}_{3}$ thin film. The cross-sectional STEM image (Fig.1 a) of the as-grown heterostructure revealed $2 \mathrm{D}$ nanochannels with $\sim 1 \mathrm{~nm}$ in thickness, penetrating through the $\mathrm{SrTiO}_{3} / \mathrm{La}_{0.7} \mathrm{Sr}_{0.3} \mathrm{MnO}_{3}$ heterostructure. Edge dislocations were found in the $\mathrm{La}_{0.7} \mathrm{Sr}_{0.3} \mathrm{MnO}_{3} / \mathrm{TbScO}_{3}$ interface. Bi atoms diffused from the top $\mathrm{Bi}$ layer through $\mathrm{SrTiO}_{3}$ and $\mathrm{La}_{0.7} \mathrm{Sr}_{0.3} \mathrm{MnO}_{3}$ layers and toward $\mathrm{La}_{0.7} \mathrm{Sr}_{0.3} \mathrm{MnO}_{3} / \mathrm{TbScO}_{3}$ interface, and $\mathrm{Mn}$ atoms diffused upward from $\mathrm{La}_{0.7} \mathrm{Sr}_{0.3} \mathrm{MnO}_{3}$ layer. It was found that the diffusion of $\mathrm{Bi}$ occurs along channels which are coincidence with the extra lattice plans of the edge dislocations located at the $\mathrm{SrTiO}_{3}$ and $\mathrm{La}_{0.7} \mathrm{Sr}_{0.3} \mathrm{MnO}_{3}$ interface. The atomic resolution EDS mapping (Fig. 1c) shows the existence of $\mathrm{Mn}$ and $\mathrm{Bi}$ atoms in the channels. In Fig. 2a, the in-plane conductive atomic force microscopy (c-AFM) indicates the nanochannels grow through the entire film. The conductivity mapping (Fig. 2b) and corresponding I-V curves (Fig. 2d) indicate that the nanochannels are conductive with diode-like behavior.

In conclusion, we report the observation of dislocation-inducced conductive 2D nanochannels in a 3D insulating oxide thin film. Further analysis on the structure and composition shows the existence of $\mathrm{BiMnO}_{3}$, which will be discussed together with more studies on transport magnetism and phase field simulations. This work opens up a way to create $2 \mathrm{D}$ conductive path in $3 \mathrm{D}$ insulator, giving raise to novel and flexible multidimensional manipulations for nanodevices [4]. 
References:

[1] A Nakamura et al., Nature Materials 2 (2003), p. 453.

[2] W Zhou et al., Science Advances 4 (3) (2018), p. eaap9096.

[3] Y Han et al., Nature Materials 17 (2017), p. 129.

[4] This work was supported by the Department of Energy (DOE) under Grant DE-SC0014430. TEM experiments were conducted using the facilities in the Irvine Materials Research Institute (IMRI) at the University of California-Irvine

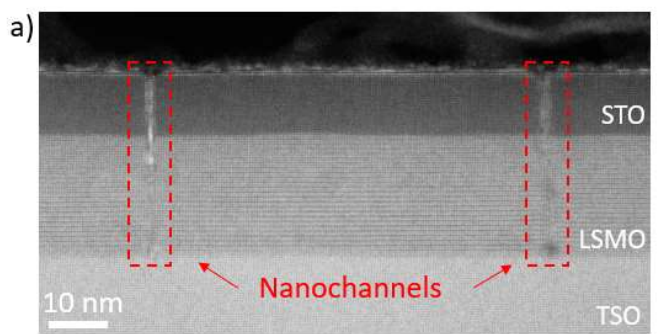

b)
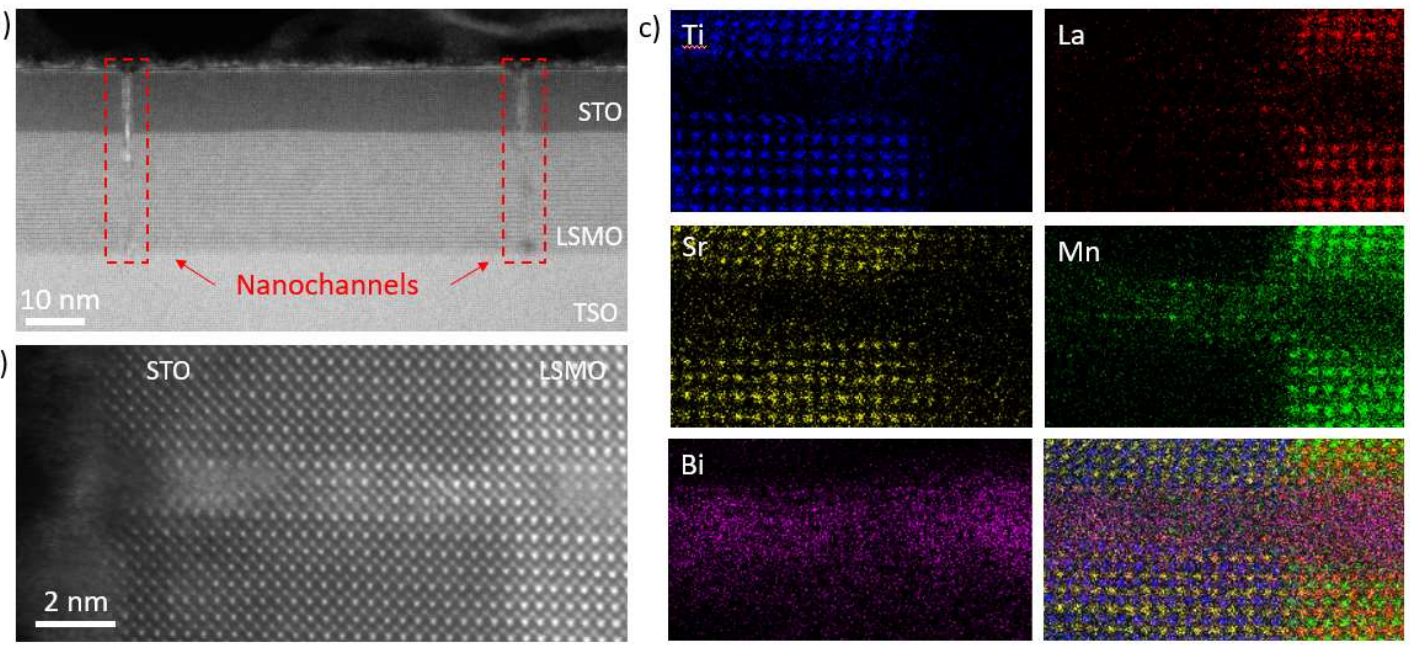

Figure 1. 2D nanochannels and corresponding atomic EDS. (a) Cross-sectional HAADF STEM image and (b) nanochannel atomic structures. (c) is the corresponding atomic EDS mapping on the nanochannel shown in (b).
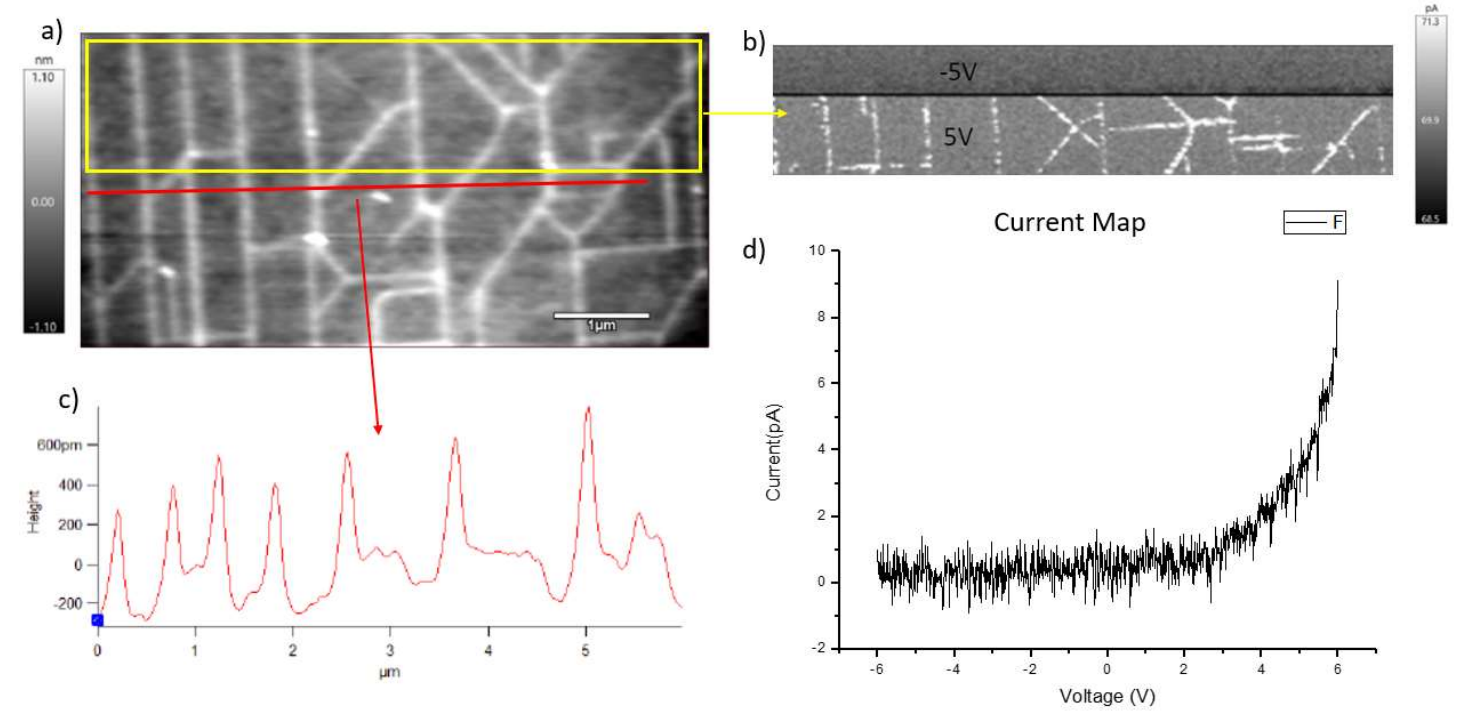

Figure 2. Height and conductivity measurements of 2D nanochannels. (a) Planner view c-AFM phase image of 2D nanochannels with (b) conductivity measurements and (d) corresponding I-V curve, and (c) height measurements. 